\title{
Anisotropy of a Tensorial Bishop's Coefficient for Wetted Granular Materials
}

\author{
Kun Wang, S.M.ASCE ; and WaiChing Sun, M.ASCE ${ }^{2}$
}

\begin{abstract}
The objective of this research is to use grain-scale numerical simulations to analyze the evolution of stress anisotropy exhibited in wetted granular matter. Multiphysical particulate simulations of unsaturated granular materials were conducted to analyze how the interactions of contact force chains and liquid bridges affect macroscopic responses under various suction pressure and loading histories. To study how the formation and rupture of liquid bridges affect the mechanical responses of wetted granular materials, a series of suction-controlled triaxial tests were conducted with two grain assemblies, one composed of large particles of similar sizes, the other composed of a mixture of large particles with significant amount of fines. The results indicate that capillary stresses are anisotropic in both sets of specimens, and that stress anisotropy is more significant in granular assemblies filled with fine particles. A generalized tensorial Bishop's coefficient is introduced to analyze the connections between microstructural attributes and macroscopic responses. Numerical simulations presented in this paper indicate that the principal values and directions of this Bishop's coefficient tensor are path dependent. DOI: 10.1061/(ASCE)EM .1943-7889.0001005. () 2015 American Society of Civil Engineers.
\end{abstract}

Author keywords: Unsaturated porous media; Young-Laplace equation; Bishop's effective stress; Capillary stress; Pendular regime; Discrete element simulation.

\section{Introduction}

Many near-surface granular materials, such as sand, silt, and seafloor sediment, are infiltrated by water and air owing to rainfall, groundwater-level changes, or external loadings that promote fluid transport. This moisture content may transform the mechanical responses of grains that are otherwise cohesionless and thus reshape the earth surface features. For instance, adding small water in cohesionless dry sand may create enough surface tensions among grains to transform dry sand into an ideal raw material for sculptures. The moisture content may also change the frictional coefficient of granular materials - a fact exploited by ancient Egyptians to facilitate the sliding of heavy objects, as evidenced by the wall painting from 1880 BCE in the tomb of Djehutihotep (Fall et al. 2014). In 1490, Leonardo de Vinci began his investigations on capillary phenomena. His study on capillaries continued with the work of Laplace, Young, Gauss, Rayleigh, and numerous others in mathematics, physics, mechanics, and engineering (Koiso and Palmer 2007). In a nearly saturated state, capillary effect is nearly isotropic. However, in funicular and pendular regimes, where water may form liquid clusters and bridges, the anisotropy of the capillary effect can be significant. The surface tension and the presence of menisci may have important implications for the mechanical responses of granular matter. In the pendular regime, where the degree of saturation is low, the intermolecular forces exerted on the solid-fluid interface may be strong enough that the liquid may stick and form a pendular bridge in between solid grains

\footnotetext{
${ }^{1}$ Graduate Student, Dept. of Civil Engineering and Engineering Mechanics, Columbia Univ., 610 SW Mudd, New York 10027.

${ }^{2}$ Assistant Professor, Dept. of Civil Engineering and Engineering Mechanics, Columbia Univ., 614 SW Mudd, New York 10027 (corresponding author). E-mail: wsun@columbia.edu

Note. This manuscript was submitted on December 31, 2014; approved on July 22, 2015; published online on September 11, 2015. Discussion period open until February 11, 2016; separate discussions must be submitted for individual papers. This paper is part of the Journal of Engineering Mechanics, (C) ASCE, ISSN 0733-9399/B4015004(15)/\$25.00.
}

instead of flow in the pores. While a liquid bridge may introduce an attractive force among grains, the relative movement of the grains connected by the liquid bridges may cause it to spread, stretch, and rupture.

At the macroscopic level, the forces due to interactions between solid grains and liquid bridges are often collectively taken into account as an isotropic suction or capillary stress exerted on a continuum mixture composed of solid, air, and liquid (Bishop 1959; Bishop and Blight 1963). This isotropic capillary pressure is often incorporated into hydromechanical finite-element models via an extension of effective stress theory. Equipped with appropriate plasticity models that replicate the suction-dependent constitutive responses [e.g., basic Barcelona model (Wheeler et al. 2002)] and retention behaviors, this macroscopic approach has enjoyed great success in modeling unsaturated porous media, especially when the degree of saturation is sufficiently high (Lewis and Schrefler 1998; Zienkiewicz et al. 1999; Borja and Koliji 2009; Sheng et al. 2004). Nevertheless, recent experimental and theoretical studies have demonstrated that capillary stress is in fact anisotropic in a pendular regime (Lu and Likos 2004; Scholtès et al. 2009). This anisotropy evolves as liquid bridges form or rupture in the deforming granular material (Richefeu et al. 2006; Scholtès et al. 2009; Wan et al. 2014). Since the migration of water inside the wetted granular materials also affects the size and shape of the liquid bridges, this anisotropic suction effect is expected to be hysteretic and depends strongly on air and water diffusion. As a result, moisture content may significantly alter how wave propagates in unsaturated granular materials. Nevertheless, the exact mechanism that leads to this evolving anisotropic effect has not been fully elucidated (Wan et al. 2014).

This study aims at using discrete element simulations to improve the understanding of the grain-air-water interaction mechanism at the grain scale and of how the micromechanical interaction induces macroscopic stress anisotropy. In particular, a linear normal contact model is used to model the normal contact, while the tangential response is governed by the Coulomb friction law. This mechanical discrete element model is coupled with a capillary 
model in which solutions of the Young-Laplace equation are used to compute the force exerted on the particles by liquid bridges. From a microscopic perspective, the menisci may form a forcechain network similar to the force chain of a grain-to-grain contact network. As the grain assemblies are subjected to external loadings, liquid bridges may form or rupture owing to the motion of particles and thus change the topology of the meniscus network. Since the rupture and formation of liquid bridges are governed by the distance between particles, grain size distribution plays an important role in the evolution of a meniscus network. To study how the suction effect varies with grain size distribution, the authors conducted a suction-controlled experiment on two grain assemblies, one composed of particles of similar sizes and the other composed of a mixture of large particles and fines. The partial Cauchy stresses are homogenized from both the solid grains and meniscus networks. Simulation results from suction-controlled compression tests at a variety of suction levels are analyzed.

As for notations and symbols, bold-faced letters denote tensors; the symbol $\cdot$ denotes a single contraction of adjacent indices of two tensors (e.g., $\boldsymbol{a} \cdot \boldsymbol{b}=a_{i} b_{i}$ or $\boldsymbol{c} \cdot \boldsymbol{d}=c_{i j} d_{j k}$ ); the symbol: denotes a double contraction of adjacent indices of tensor of rank two or higher (e.g., $\boldsymbol{C}: \boldsymbol{\epsilon}^{\boldsymbol{e}}=C_{i j k l} \epsilon_{k l}^{e}$ ); the symbol $\otimes$ denotes a juxtaposition of two vectors (e.g., $\boldsymbol{a} \otimes \boldsymbol{b}=a_{i} b_{j}$ ) or two symmetric second-order tensors [e.g,. $\left.(\boldsymbol{\alpha} \otimes \boldsymbol{\beta})=\alpha_{i j} \beta_{k l}\right]$.

\section{Generalized Bishop's Coefficient Tensor}

In geotechnical engineering and geomechanics practice, air-waterparticle interactions are rarely modeled explicitly. Rather, macroscopic homogenized theory, such as Biot's poroelasticity theory or mixture theory, is often used to model the collective responses of the air, water, and solid constituents at the macroscopic scale. In this situation, Cauchy stress (or other appropriate stress measures) is partitioned into three parts, i.e., the effective stress and the pore water and air pressures. Such a macroscopic treatment is often referred to as the effective stress principle. The effective stress principle was originally proposed in 1936 for saturated porous media (Terzaghi 1996; De Boer 1996; Sun 2013; Sun et al. 2013b, 2014). Terzaghi's effective stress principle may be stated in the form of two propositions:

1. Effective stress is the exclusive cause of the change in volume and shear strength of a soil; and

2. The effective stress of a soil is defined as the difference between the total applied stress and the pore fluid pressure.

Extension of the effective stress principle to unsaturated porous media has been proposed by Bishop (1959). Bishop's effective stress, which is presented in Eq. (1), is commonly accepted by the geomechanics research community, although the validity of the total stress partition between suction and effective stress is the subject of a longstanding debate and sometimes misunderstood, as indicated by Jennings and Burland (1962) and Laloui and Nuth (2009):

$$
\sigma_{i j}^{\prime}=\sigma_{i j}-p_{a} \delta_{i j}+\chi s \delta_{i j}
$$

where $\sigma_{i j}=$ total stress tensor; $s=p_{a}-p_{w}=$ difference between the pore air pressure $p_{a}$ and the pore water pressure $p_{w}$, i.e., capillary pressure; and $\chi=$ Bishop's coefficient, which is related to the degree of saturation $S_{r}$ (Bishop 1959). The major result of using Bishop's effective stress is that it enables a simple transition to Terzaghi's effective stress by imposing $\chi=S_{r}=1$ when the porous medium becomes fully saturated and $\chi=S_{r}=0$ when the porous medium is dry. Bishop's coefficient is considered to be a scalar owing to the underlying assumption that the capillary effect is isotropic. However, this assumption is not valid according to experiments conducted on wetted granular matter in the pendular regime in which the anisotropic nature of capillary effects cannot be neglected (Lu et al. 2007; Manahiloh and Muhunthan 2012; Fall et al. 2014). A possible explanation of the anisotropic effect is that the anisotropy stems from microstructural attributes. In particular, since the orientation of the force chains formed by both the granular contacts and pore water menisci are both nonisotropic, the macroscopic capillary stress, which is a function of force and branch vectors, is also not necessarily isotropic. Furthermore, since the spatial distributions of granular contacts and pore water menisci evolve when the wetted granular assemblies are subjected to external loadings, this anisotropic effect is likely to evolve according to the load history, as evidenced by experimental data obtained from computed tomography (CT) images, (Alonso et al. 2010; Hicher and Chang 2007; Hashemi et al. 2013; Higo et al. 2013). Moreover, Richefeu et al. (2006), Soulie et al. (2006), and Scholtès et al. (2009) conducted discrete element simulations to investigate contact and liquid bridge fabric. They showed that liquid bridge fabric is both anisotropic and path dependent when subjected to shear loading. This anisotropy may lead to the anisotropy of macroscopic effective and capillary stress tensors (obtained from homogenizing grain-tograin and grain-water-grain contact force and branch vectors) being anisotropic. In this study, the authors' objective is to quantify the interplay between the microscopic force-chain orientation and macroscopic anisotropy of effective and capillary stresses. In particular, the macroscopic stress is obtained via homogenization of force and branch vectors (Sun et al. 2013a; Iwashita and Oda 2000; Cambou et al. 2009), i.e.,

$$
\begin{gathered}
\sigma_{i j}=\sigma_{i j}^{\text {contact }}+\sigma_{i j}^{\text {capillary }}+p_{a} \delta_{i j} \\
\sigma_{i j}^{\text {contact }}=\sigma_{i j}^{\prime}=\frac{1}{V} \sum_{g c=1}^{N_{g c}} F_{\text {contact }, i}^{g c} l_{j}^{g c} \\
\sigma_{i j}^{\text {capillary }}=\frac{1}{V} \sum_{n=1}^{N_{n}} F_{\text {capillary }, i}^{n} l_{j}^{n}
\end{gathered}
$$

where $g c$ denotes grain contact and $n$ denotes liquid bridge neck. $l^{g c}$ and $l^{n}$ represent the vectors connecting the centers of two grains corresponding to the solid contact and liquid meniscus, respectively. Since the microstructural attributes of both force and branch vectors depend on the orientations of particle contacts, the capillary stress $\sigma_{i j}^{\text {capillary }}$, which is the sum of dyadic products of capillary forces and branch vectors as defined in Eq. (2), is usually not isotropic, even though the macroscopic suction remains isotropic. As a result, the authors introduce a tensorial form of Bishop's coefficient to replace the isotropic term $\chi s \delta_{i j}$ in Eq. (1) by the tensorial form $\chi_{i j} s$ :

$$
\sigma_{i j}^{\prime}=\sigma_{i j}-p_{a} \delta_{i j}+\chi_{i j} s
$$

Comparison of Eqs. (5) and (2) leads to

$$
\sigma_{i j}^{\text {capillary }}=-\chi_{i j} s=-\chi_{i j}\left(p_{a}-p_{w}\right)
$$

Like the isotropic classical counterpart in Laloui and Nuth (2009), this generalized Bishop's coefficient tensor $\chi_{i j}$ is generally not a constant parameter but depends on microstructural attributes, such as force chain topology and pore size distribution. These features will be analyzed in the following sections in which suction-controlled triaxial loadings are prescribed in wetted 
granular assemblies. The authors account for these changes by micromechanical studies of contact normal and meniscus orientation as well as interparticle distance. The main goal is to investigate how the degree of anisotropy of $\chi_{i j}$ evolves differently in wetted granular materials of different grain size distributions. The effect of fines content and suction pressure on the principal values and directions of $\chi_{i j}$ will also be examined.

\section{DEM Models}

The behavior of wet granular media under external loads has been studied using both experimental and numerical approaches (Bishop and Blight 1963; Fall et al. 2014; Laloui and Nuth 2009; Wheeler et al. 2002). Because the overall behavior of a specimen is strongly related to the interaction between individual particles and liquid bridges, understanding the microscopic forces, grain structures, and water distribution is a key step toward understanding the macroscopic behavior of unsaturated porous media. While recent advances in tomographic imaging techniques may potentially open the door to studying the connection between microstructures and macroscopic responses, applying this technique to wetted granular matter remains a technically challenging task Hashemi et al. (2013). Meanwhile, macroscopic continuum models based on either mixture theory or extensions of Biot's poroelasticity theory have often been used to capture the mechanical behaviors of wetted granular matter. In macroscopic continuum models, air-water-solid interactions are not modeled explicitly. Rather, they are collectively considered as a continuum mixture, with each constituent (solid, water, and air) occupying a fraction of the volume at each macroscopic material point (Borja and Koliji 2009; Laloui and Nuth 2009; Salager et al. 2006; Sun et al. 2011a, b).

An alternative to this continuum approach is to explicitly model the interactions among particles, liquid bridges, and air in wetted granular assemblies. This discrete mechanics approach, often referred to as the distinct or discrete element method (DEM), was first introduced by Cundall and Strack (1979). In DEM, the motions of particles are governed by Newton's second law, and their positions and velocities are computed by an explicit finite difference integration scheme. In this study, an open-source discrete element code, yet another dynamic engine (YADE), is used to model the friction-cohesive mechanical behavior of wetted granular matter in a pendular regime (Šmilauer et al. 2014). The linkage between microscale and macroscale properties is established by the homogenization techniques, where macroscopic strain and stress tensors of a representative elementary volume are derived from the positions of particles and liquid bridges, as well as the normal, tangential forces exerted on them (Kruyt and Rothenburg 1996). The capillary physics was introduced into the model by solving the Young-Laplace equation. Various numerical simulations have been conducted to examine the evolution of mechanical and hydraulic properties of unsaturated granulates. To simplify the problem, the authors assume that the degree of saturation remains low enough during the numerical simulations such that water only exists in the form of liquid bridges between the surface of grain pairs. As a result, the interparticle normal force represents a combined effect of the grain-to-grain normal contact force and the capillary force developed in liquid bridges. Such a hypothesis is usually valid at low water content, and the corresponding unsaturated state is called the pendular regime. Triaxial compression tests were performed on numerical samples, and the effect of partial saturation on macro and micro behaviors was studied.

\section{Contact Friction Model}

The classic frictional-normal contact law for cohensionless particles is adopted in the DEM simulation to model the granular contact between two particles. Contact force between two particles is generated when the two overlap each other and follows Hooke's law. Two stiffnesses can be defined: $K_{n}$ and $K_{t}$ in the normal $\boldsymbol{n}$ and tangential direction $t$ of the contact plane, respectively, as shown in Fig. 1. Incremental changes in the normal force $d \boldsymbol{F}_{n}$ and the tangential force $d \boldsymbol{F}_{t}$ are thus expressed as

$$
\begin{gathered}
d \boldsymbol{F}_{n}=K_{n} d U_{n} \boldsymbol{n} \\
d \boldsymbol{F}_{t}=-K_{t} d \boldsymbol{U}_{t}
\end{gathered}
$$

where $d U_{n}=$ incremental normal overlap distance and $d \boldsymbol{U}_{t}=$ incremental tangential relative displacement vector.

The tangential force is limited by a maximum value of $\left\|\boldsymbol{F}_{t}^{\max }\right\|=-\mu\left\|\boldsymbol{F}_{n}\right\|$ according to Coulomb's friction law, where $\mu$ is the Coulomb friction coefficient. It is related to the interparticle friction angle $\phi_{c}$ by $\mu=\tan \left(\phi_{c}\right)$.

More complex models, such as Hertz-Mindlin, could also be used (Sun et al. 2013a), yet some studies suggest that there are no significant improvements compared to the linear model and that the calculation time increases significantly Di Renzo and Di Maio (2004).

\section{Capillary Model}

Many DEM liquid bridge models have been developed to introduce attractive capillary forces between grains in a pendular regime, and some of them are based on solving the Young-Laplace equation. For example, some researchers approximate the form of a liquid bridge by a torus and derive the water volume and capillary force from the suction pressure $\Delta p=p_{\text {air }}-p_{\text {water }}$, but the capacity to treat polydisperse assemblies is limited (Jiang et al. 2004). There are also models that explicitly express the capillary force in terms of the geometric parameters of a liquid bridge, but in those cases the suction pressure becomes an additional unknown (Soulie et al. 2006). In this study, the authors employ the capillary model implemented in the DEM code YADE to simulate the capillary effect at the grain scale. This model, originally introduced by

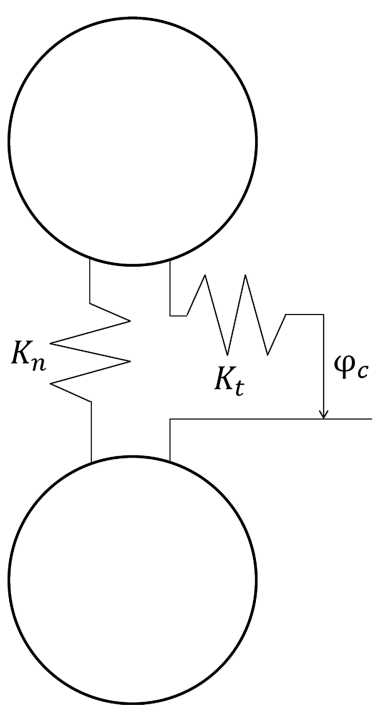

(a)

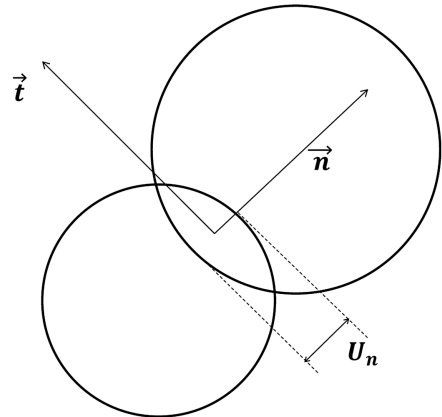

(b)
Fig. 1. Contact friction model 
Scholtès et al. (2009), differs from the aforementioned approaches in that the suction pressure $\Delta p$ is assumed to be uniform in space and serves as the control parameter for unsaturated sample tests. The distribution of liquid bridges in the samples is hence almost homogeneous. The meniscus volumes and capillary forces are directly computed from $\Delta p$ and grain-pair geometry by interpolating discrete numerical solutions of the Young-Laplace equation. The authors briefly outline the capillary model in this section, and interested readers are referred to Scholtès et al. (2009) for further information.

The geometry and parameters of the liquid bridge model are illustrated by Fig. 2. The complex profile of the menisci is described by the curve function $y(x)$ in the coordinate system of Fig. 2. The two principal radii of the local surface curvature are given by $r_{1}(x)=y(x) \sqrt{1+y^{\prime 2}(x)}$ and $r_{2}(x)=\left[1+y^{\prime 2}(x)\right]^{3 / 2} /$ $y^{\prime \prime}(x)$. The curvature of liquid bridge $\mathrm{C}$ is defined by $1 / C=$ $1 / r_{1}+1 / r_{2}, R_{1}$ and $R_{2}$ are sphere radii, $\zeta_{1}$ and $\zeta_{2}$ are filling angles, $\theta$ is the wetting angle, $x_{c 1}$ and $x_{c 2}$ are the $x$-coordinates of the solid-liquid-air interface, and $D$ is the intergranular distance.

The liquid bridge model is governed by a system of four nonlinear equations. First, the curvature of liquid bridge $C$ can be related to the suction pressure $\Delta p=p_{\text {air }}-p_{\text {water }}$ and the surface tension $\gamma$ through the Young-Laplace equation

$$
\Delta p=s=\frac{\gamma}{C}
$$

The nonlinear differential equation for the meniscus profile $y(x)$ is therefore written as follows:

$$
\frac{\Delta p}{\gamma}\left[1+y^{\prime 2}(x)\right]^{3 / 2}+\frac{1+y^{\prime 2}(x)}{y(x)}-y^{\prime \prime}(x)=0
$$

The attractive capillary force exerted by the liquid bridge can be estimated at the profile neck $y_{0}$ based on the so-called gorge method (Hotta et al. 1974):

$$
F_{\text {capillary }}=2 \pi y_{0} \gamma+\pi y_{0}^{2} \Delta p
$$

The corresponding volume of the liquid bridge between two spheres is computed by

$$
\begin{aligned}
V= & \pi \int_{x_{c 1}}^{x_{c 2}} y^{2}(x) d x-\frac{1}{3} \pi R_{1}^{3}\left[1-\cos \left(\zeta_{1}\right)\right]^{2}\left[2+\cos \left(\zeta_{1}\right)\right] \\
& -\frac{1}{3} \pi R_{2}^{3}\left[1-\cos \left(\zeta_{2}\right)\right]^{2}\left[2+\cos \left(\zeta_{2}\right)\right]
\end{aligned}
$$

and the intergranular distance $D$

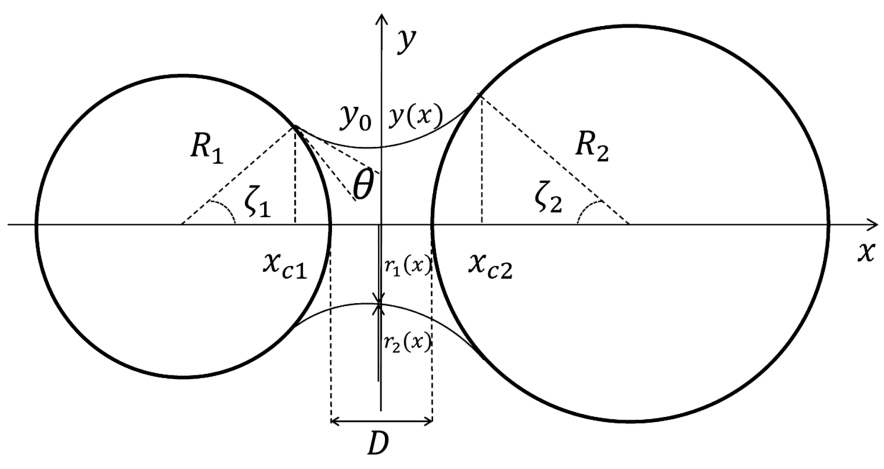

Fig. 2. Liquid bridge model

$$
D=R_{2}\left[1-\cos \left(\zeta_{2}\right)\right]+x_{c 2}+R_{1}\left[1-\cos \left(\zeta_{1}\right)\right]-x_{c 1}
$$

The solution scheme of the foregoing system of Eqs. (10)-(13) incorporated into YADE leads to a suction-controlled model where the capillary force and volume of a liquid bridge can be determined by the imposed macroscopic suction pressure $\Delta p$ for a given pair of spheres of radius $R_{1}$ and $R_{2}$ and their intergranular distance $D$. The formation of a liquid bridge is assumed to occur once the two grains come into contact. The critical intergranular distance beyond which the liquid bridge ruptures is not explicitly given but corresponds to the distance value from which the Young-Laplace equation no longer provides a stable liquid bridge (Lian et al. 1993). The distribution of liquid bridges evolves with the updated sample configuration, and the overall degree of saturation is directly computed using the total volume of menisci. In this study, the surface tension is set at $0.073 \mathrm{~N} / \mathrm{m}$, and the contact angle $\theta$ is equal to 0 in the program.

The current model presents some simplifications and limitations. It is assumed that all particles in the granular assemblies are spherical, and surface roughness and gravity are negligible (Toker et al. 2014). The degree of saturation is restricted to low values $(\mathrm{Sr}<12 \%)$, so that it is assumed that overlappings of neighboring interparticle liquid bridges are negligible. The interparticle friction angle is assumed to be constant and not to vary with the degree of saturation. The model also does not incorporate the redistribution law of water when the menisci break (Shi and McCarthy 2008).

\section{DEM Experimental Results}

\section{Evolution of Bishop's Coefficient during Suction- Controlled Triaxial Tests}

The researchers first investigate the evolution of Bishop's effective stress coefficient $\chi$ during a triaxial compression test on a specimen of equal-sized spheres. The diameter of the grains is set to a small value of $0.1 \mathrm{~mm}$ (fine sand). A box made of six rigid frictionless

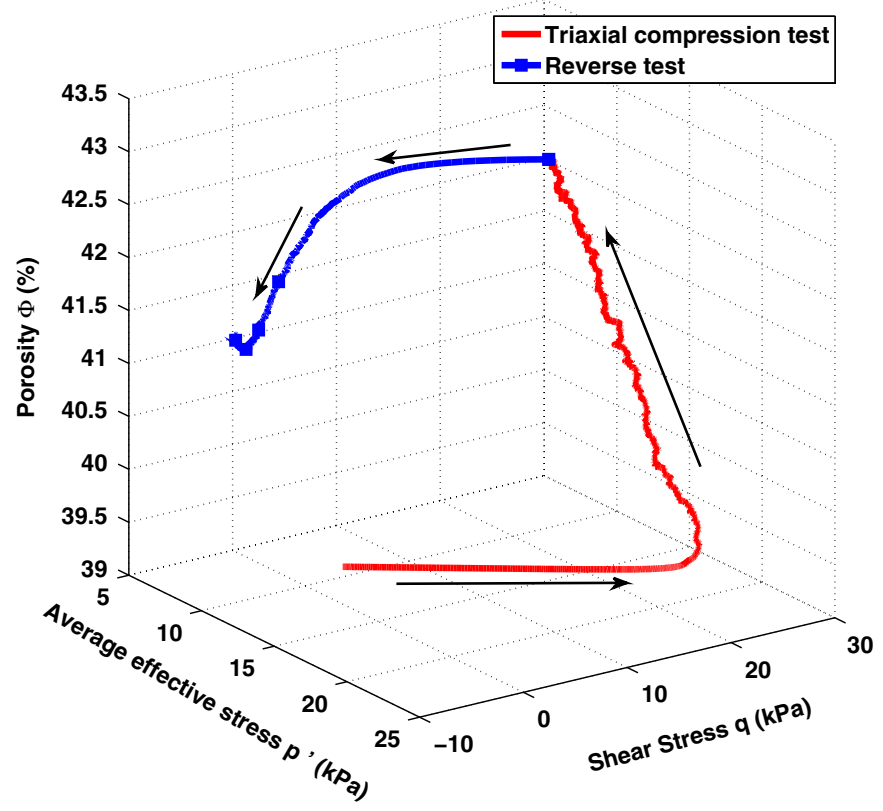

Fig. 3. State path of triaxial compression test and reverse test; arrows point in loading direction 
walls is constructed, inside which a DEM assembly is prepared by a sphere packing generation algorithm available in YADE and contains approximately 11,000 particles. The material parameters are chosen to be the same as the previous DEM experiments in Scholtès et al. (2009) (Young's modulus $E=50 \mathrm{MPa}$, ratio of tangential to normal stiffness $\alpha=k_{t} / k_{n}=0.5$, and friction angle $\phi_{c}=30^{\circ}$ ). The deformation of the DEM sample is carried out by moving the walls according to the prescribed loading processes. The suction pressure in the numerical test is maintained constant $(10 \mathrm{kPa})$, corresponding to a suction-controlled triaxial test on unsaturated soil. This first simulation consists of three stages. An isotropic compression takes place under a confining pressure of $10 \mathrm{kPa}$. The quasi-static compression is carried out along the Y-axis up to $15 \%$ axial strain, at which point the sample is near its critical state, while the lateral borders ( $\mathrm{X}, \mathrm{Z}$ axis) always provide a $10 \mathrm{kPa}$ confining pressure. The deformed sample is then subjected to an extensive loading path along the Y-axis back to its original zero-strain configuration in order to determine whether the evolution of Bishop's coefficient will follow the same curve in the opposite direction. The state path of the numerical test is
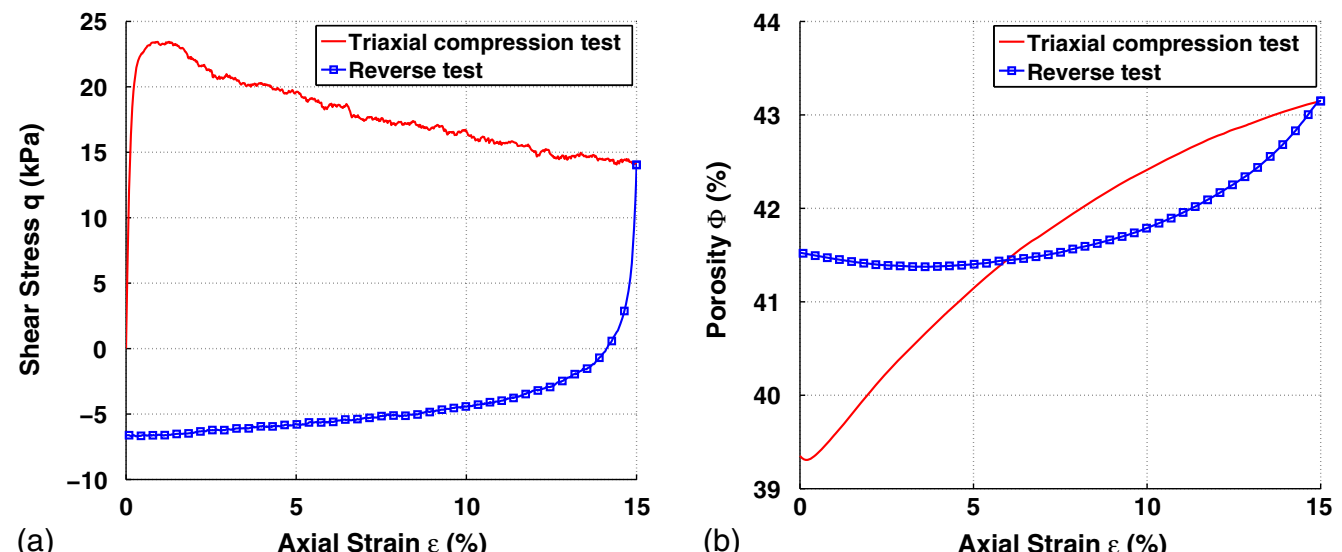

(a)

Axial Strain $\varepsilon(\%)$

(b)

Axial Strain $\varepsilon(\%)$
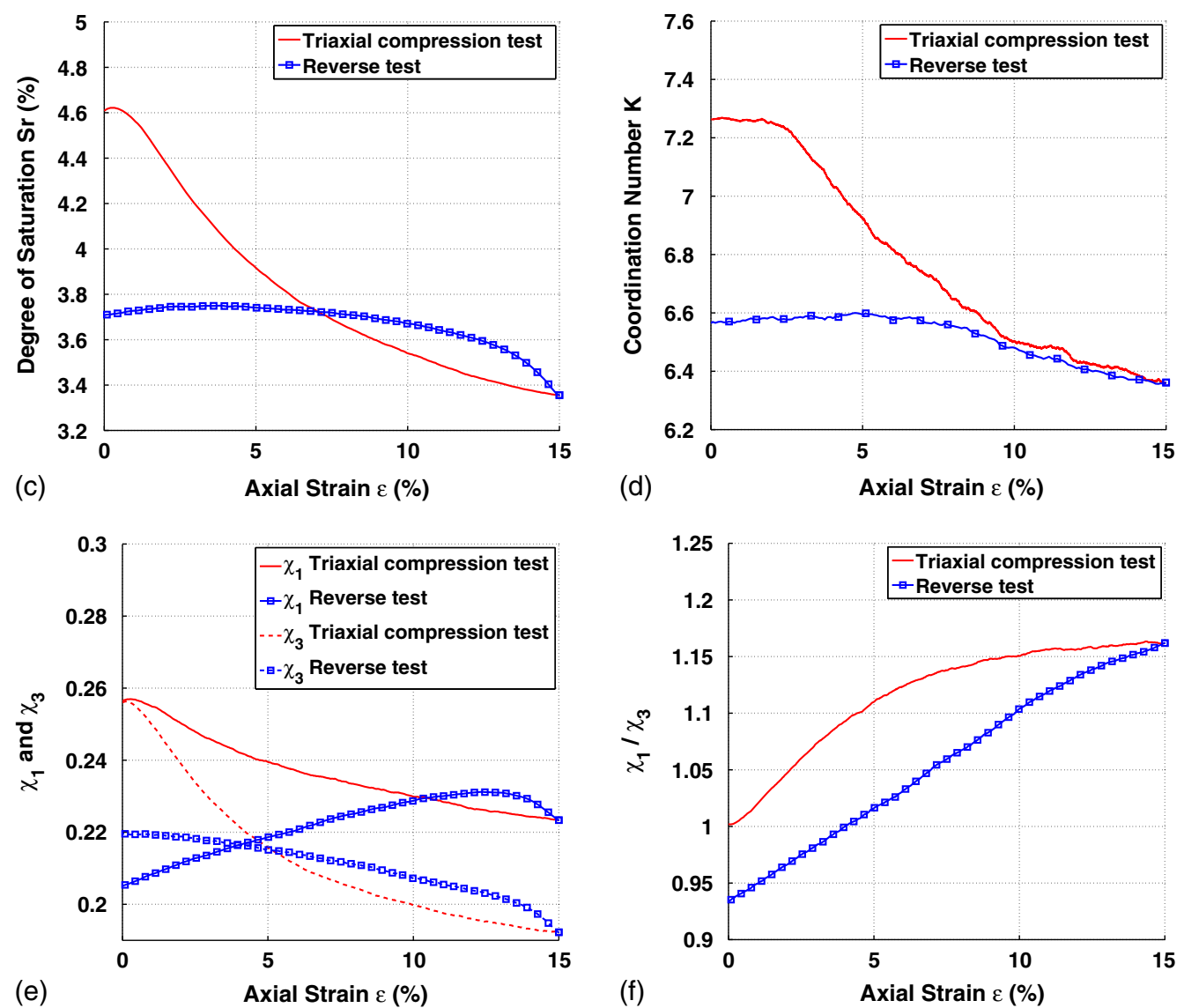

Fig. 4. Evolution of the (a) shear stress in the triaxial compression test (left to right) and the reverse extension test (right to left); (b) porosity in triaxial compression test (left to right) and reverse extension test (right to left); (c) saturation in triaxial compression test (left to right) and reverse extension test (right to left); (d) coordination number in triaxial compression test (left to right) and reverse extension test (right to left); (e) major and minor principal values of Bishop's coefficient in triaxial compression test (left to right) and reverse extension test (right to left); (f) ratio of major and minor principal values of Bishop's coefficient in triaxial compression test (left to right) and reverse extension test (right to left) 
shown in Fig. 3 in the space of the shear stress $q=\sigma_{1}-\sigma_{3}$, the average effective stress $p^{\prime}=1 / 3\left(\sigma_{1^{\prime}}+\sigma_{2^{\prime}}+\sigma_{3^{\prime}}\right)$, and the porosity $\Phi$, where subscripts 1,2 , and 3 denote the major, intermediate, and minor principal directions in the compression test, respectively. Note that the authors conserve this assignment, although at the late phase of the reverse test the major and minor principal directions will switch between them $\left(\sigma_{1}<\sigma_{3}\right)$, thereby allowing the shear stress to be negative and the ratio of the principal values to be smaller than 1 .

The simulated hydromechanical responses, such as the shear stress and the porosity (Fig. 4) resemble those in classical triaxial compression and extension tests on sand (Shapiro 2000). In the compression test, as expected, the preconsolidated sample shows an initial contractancy and then dilates with a decreasing slope until a critical porosity or degree of saturation is reached. During the extension test, the sample is first compressed and then dilated. However, the path it follows does not coincide with the previous curves in the compression test, although the only change in the experimental conditions is the reverse of the axial loading direction. This can be foreseen based on the plastic nature of granular materials: the deformation of granular media induces a permanent change in the contact fabric because the internal contact and liquid bridge forces between grains are not capable of restoring the initial configuration. Under the external restoring load, the total major principal stress $\sigma_{1}$ quickly drops to the controlled lateral stress $\sigma_{3}$ at $14.2 \%$ strain (with initial isotropically confined sample as reference), and the principal directions alter thereafter. The switch between the contractancy and dilatancy occurs at $4 \%$ strain, as indicated by the porosity $\Phi$, the degree of saturation $S_{r}$, and the coordination number $K$. The authors distinguish between the coordination number of grain contact forces and the coordination number of liquid bridge forces and present their evolution in Fig. 5. The number of grain contacts immediately decreases when the deviatoric loading begins, and the evolution of the number of liquid bridges lags behind since a meniscus could persist when a grain pair initially in contact is separated by a distance smaller than the model's rupture distance.

During the compression test (Fig. 4), both the major principal component $\chi_{1}$ and the minor component $\chi_{3}$ of $\chi$ briefly increase and then decrease smoothly. The fact that $\chi_{3}$ decreases more rapidly than $\chi_{1}$ clearly reveals the anisotropic nature of Bishop's effective stress coefficient. The measure of the anisotropy $\chi_{1} / \chi_{3}$ also closely follows the same pattern, suggesting a relation between the density of the sample and the anisotropy of the capillary stress tensor.

As for the extension test shown in Fig. $4, \chi_{1}$ and $\chi_{3}$ exhibit different behaviors: $\chi_{1}$ rises to a peak value at $12.5 \%$ strain whereas
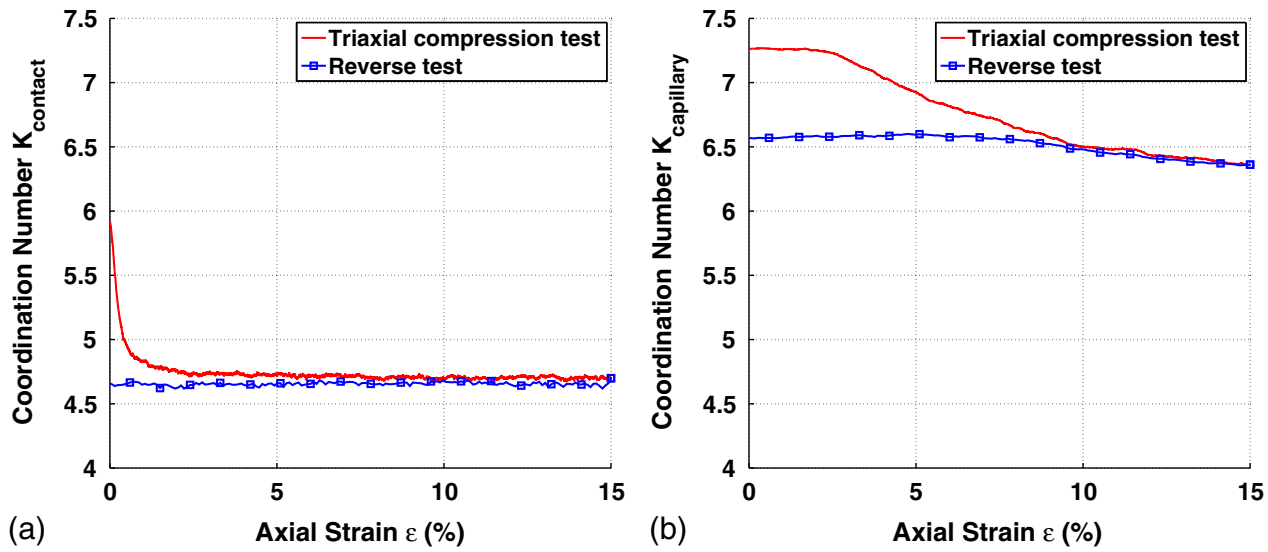

Fig. 5. Evolution of coordination number of (a) grain contact forces in triaxial compression test (left to right) and reverse extension test (right to left); (b) liquid bridge forces in triaxial compression test (left to right) and reverse extension test (right to left)
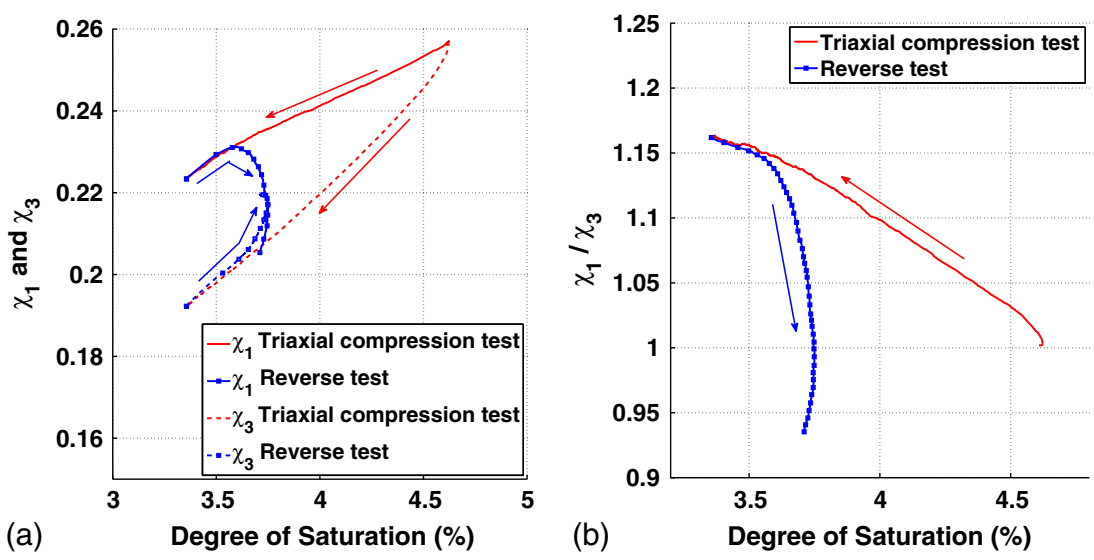

Fig. 6. (a) Major and minor principal values $\chi_{1}$ and $\chi_{3}$ of Bishop's coefficient versus degree of saturation; (b) ratio of major and minor principal values $\chi_{1}$ and $\chi_{3}$ of Bishop's coefficient versus degree of saturation; at the late phase of the reverse test, the major and minor principal directions switch between them, yet Subscripts 1 and 3 still refer to the same principal directions as before, allowing the ratio to be smaller than 1 


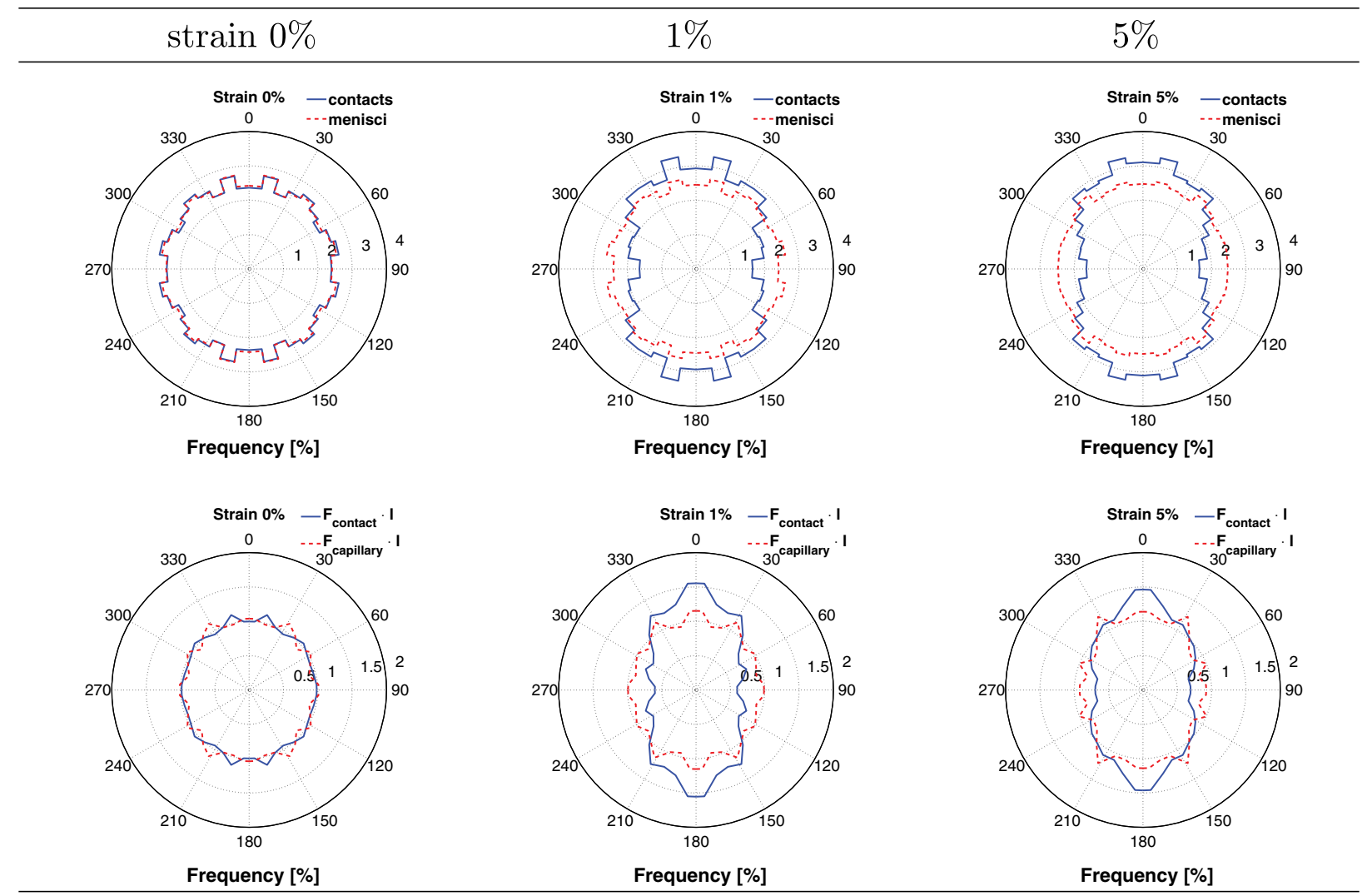

Fig. 7. Distribution of contact and menisci normal, $P_{\text {contact }}(\boldsymbol{n})$ and $P_{\text {meniscus }}(\boldsymbol{n})$, and average force intensity, $\left\langle\mathbf{F}_{\text {contact }} \cdot \boldsymbol{l}\right\rangle_{\boldsymbol{n}}$ and $\left\langle\mathbf{F}_{\text {capillary }} \cdot \boldsymbol{l}\right\rangle_{\boldsymbol{n}}$, during suction-controlled triaxial compression test

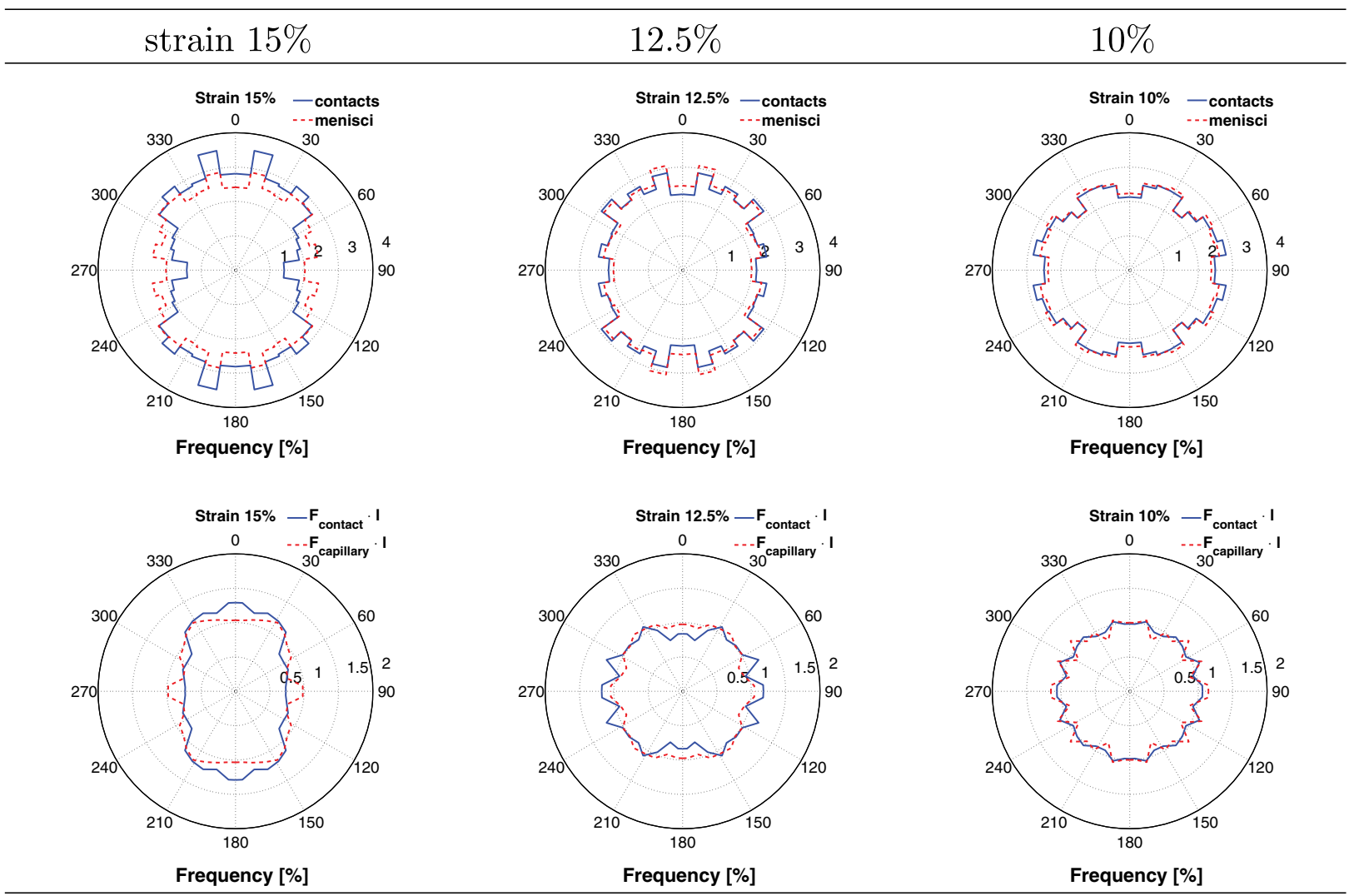

Fig. 8. Distribution of contact and meniscus normal, $P_{\text {contact }}(\boldsymbol{n})$ and $P_{\text {meniscus }}(\boldsymbol{n})$, and average force intensity, $\left\langle\mathbf{F}_{\text {contact }} \cdot \boldsymbol{l}\right\rangle_{\boldsymbol{n}}$ and $\left\langle\mathbf{F}_{\text {capillary }} \cdot \boldsymbol{l}\right\rangle_{\boldsymbol{n}}$, during suction-controlled triaxial extension test 


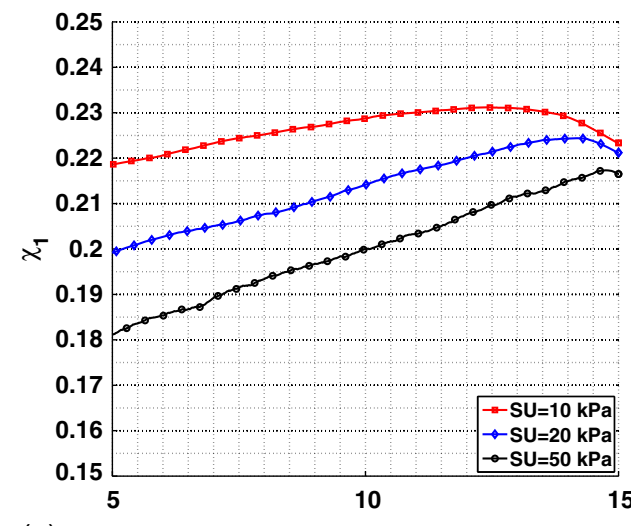

(a)

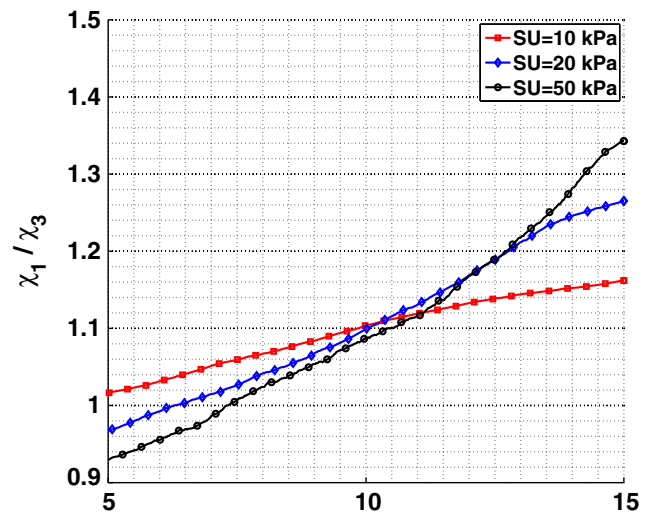

(b)

Axial Strain $\varepsilon(\%)$
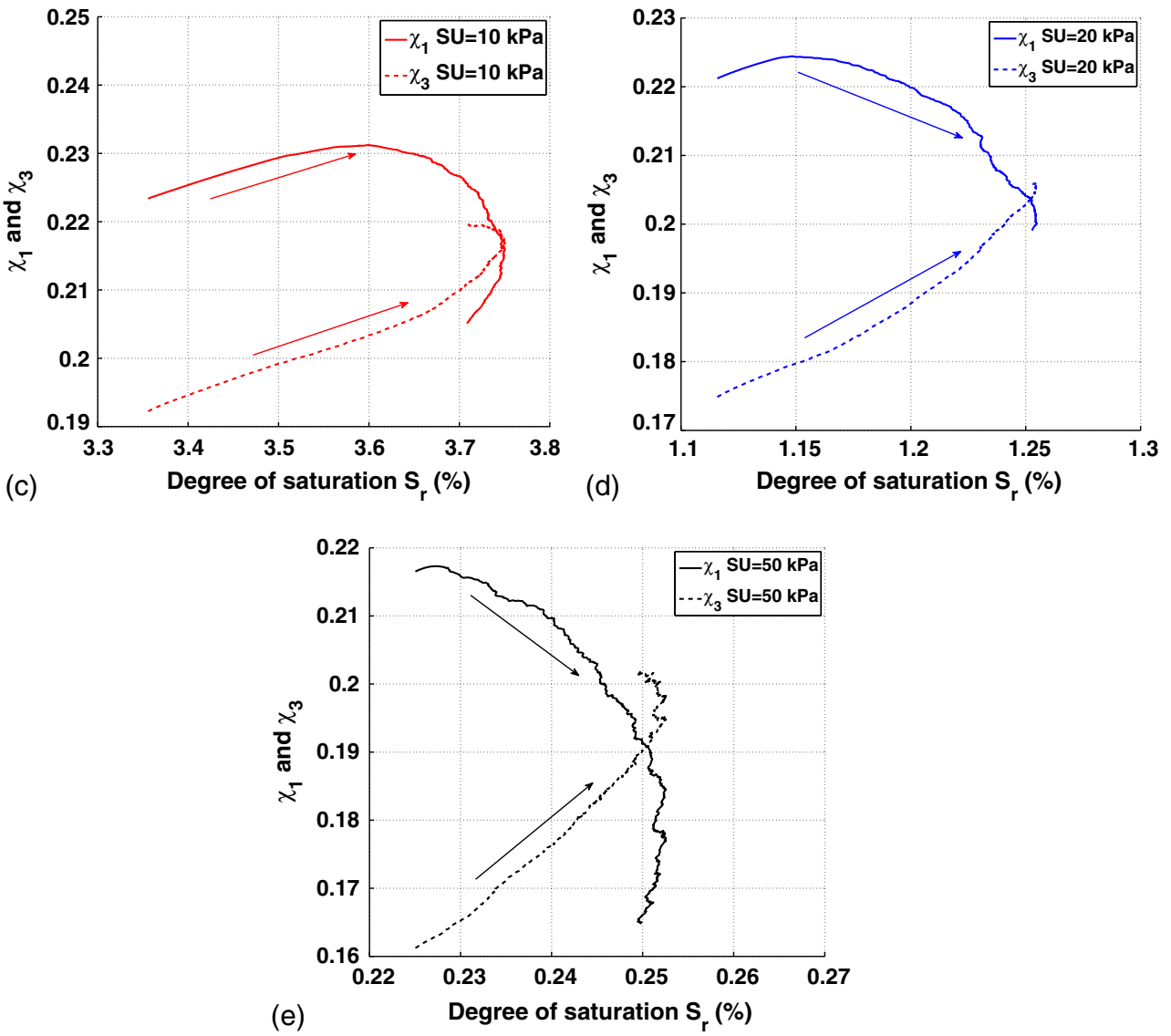

Fig. 9. Evolution of Bishop's coefficient during extension test (from 15 to $5 \%$ ): (a) $\chi_{1}$ versus axial strain; (b) $\chi_{1} / \chi_{3}$ versus axial strain; (c) $\chi_{1}$ and $\chi_{3}$ versus degree of saturation $S_{r}$ under suction of $10 \mathrm{kPa}$; arrows point in loading direction; initial isotropic configuration is set to be the reference; (d) $\chi_{1}$ and $\chi_{3}$ versus degree of saturation $S_{r}$ under suction of $20 \mathrm{kPa}$; arrows point in loading direction; initial isotropic configuration is set to be the reference; (e) $\chi_{1}$ and $\chi_{3}$ versus degree of saturation $S_{r}$ under suction of $50 \mathrm{kPa}$; arrows point in loading direction; initial isotropic configuration is set to be the reference

$\chi_{3}$ decreases, neglecting the continuous reduction of $\Phi$ and the augmentation of $S_{r} ; \chi_{3}$ persistently increases, although both $\Phi$ and $S_{r}$ attain their extrema and evolve in opposite directions. The resulting evolvement ratio $\chi_{1} / \chi_{3}$ consists of two straight lines of different slopes [Fig. 4(f) guidelines] divided at the $12.5 \%$ strain, where $\chi_{1}$ reaches its peak.

The relation between $\chi$ and $S_{r}$ is illustrated in Fig. 6. Unlike the former totally different paths in the spaces, such as $\left(q, p^{\prime}, \Phi\right),(q, \epsilon)$, and $(\Phi, \epsilon)$, the opposite loading initially reverses $\chi_{1}, \chi_{3}$, and $\chi_{1} / \chi_{3}$ approximately along the same paths as those in the compression test. The paths all bifurcate around $S_{r}=3.58 \%$ (Fig. 6 guidelines), corresponding to the peak of $\chi_{1}$ at $12.5 \%$ strain. Bishop's coefficient becomes increasingly isotropic as its two principal components approach each other, and an unstable isotropic state is attained when $S_{r}$ reaches its extremum at $4 \%$ strain. Subsequently, the principal directions of $\chi$ alter and the degree of anisotropy rises again.

A micromechanical analysis method has been developed and is used to investigate the internal structure of liquid bridges of wet granular media. The method separates the distribution of the meniscus orientation and the distribution of the mean capillary force intensity by writing the capillary stress in the following form (Scholtès et al. 2009): 


$$
\sigma^{\text {capillary }}=\frac{1}{V} \int_{V}\left\langle\mathbf{F}_{\text {capillary }} \cdot \mathbf{l}\right\rangle_{\mathbf{n}} P_{\text {meniscus }}(\mathbf{n}) \mathbf{n} \otimes \mathbf{n} d V
$$

where $\mathbf{n}=$ unit vector of an azimuthal direction emanating from the center of the granular sample; $P_{\text {meniscus }}(\mathbf{n})=$ probability that a meniscus will have orientation $\mathbf{n}$; and $\left\langle\mathbf{F}_{\text {capillary }} \cdot \mathbf{l}\right\rangle_{\mathbf{n}}=$ mean value of the dot product of the capillary force and the branch vector along the direction $\mathbf{n}$.

Fig. 7 recapitulates the rose diagram within the YZ-plane of the distribution of $P(\mathbf{n})$ and $\langle\mathbf{F} \cdot \mathbf{l}\rangle_{\mathbf{n}}$ for both contacts and liquid bridges, setting the compression axis $\mathrm{Y}$ as the $0^{\circ}$ reference. $P_{\text {contact }}(\mathbf{n})$ increases quickly in the Y-direction and reduces in the lateral direction, in accordance with the external loading. $P_{\text {meniscus }}(\mathbf{n})$, however, has its own fabric and aligns with the Y-axis very smoothly since liquid bridges can still connect two separated grains within a rupture distance. On the other hand, both $\left\langle\mathbf{F}_{\text {contact }} \cdot \mathbf{l}\right\rangle_{\mathbf{n}}$ and $\left\langle\mathbf{F}_{\text {capillary }} \cdot \mathbf{l}\right\rangle_{\mathbf{n}}$ suddenly attain their maximum anisotropic patterns and then gradually reduce to a smaller area when the whole sample becomes loose.

With regard to the subsequent extension test, Fig. 8 illustrates the internal structure evolution when the active border moves in the opposite direction. The elevation of $\chi_{1}$ at the beginning appears to be dominated by the fact that more liquid bridges are oriented around the Y-axis. This trend stops at $12.5 \%$ strain, and $P_{\text {meniscus }}(\mathbf{n})$ starts to increase in the perpendicular direction. According to the capillary model introduced in the section "Capillary Model," the magnitude of the capillary force decreases as the intergranular distance increases. The $\langle\mathbf{F} \cdot \mathbf{l}\rangle_{\mathbf{n}}$ diagrams thus indicate that the interparticle distances along the Y-axis are slowly enlarged, and the distances along the Z-direction are reduced. The evolution of both $P_{\text {meniscus }}(\mathbf{n})$ and $\langle\mathbf{F} \cdot \mathbf{l}\rangle_{\mathbf{n}}$ results in a decrease in the major principal component of the capillary stress $\sigma_{1}^{\text {capillary }}$, and $\chi_{1}$ drops gradually, as shown in Figs. 4 and 6.

The foregoing micromechanical analysis emphasizes that the distributions of liquid bridge orientation and intergranular distance have important roles in the anisotropy of Bishop's effective stress coefficient $\chi$. The fabric of the meniscus orientation and that of intergranular distances are generally different, and their evolution under external loads depends on the previous configuration of the granular structure or the loading history. The main source of the discrepancy between contact and capillary stress anisotropies is the different role capillary interactions play in grain assemblies. Unlike solid contacts between grains, liquid bridges are capable of connecting closely separated neighboring particles and provide attractive capillary forces.

Additional simulations analogous to the previous tests were subsequently performed to study the effect of suction pressure (SU) on the evolution of $\chi$ during the extension test. The curves shown in Fig. 9 confirm that the formerly observed evolution trends of $\chi_{1}$, $\chi_{3}$, and $\chi_{1} / \chi_{3}$ are also present for $\mathrm{SU}=20 \mathrm{kPa}$ and $\mathrm{SU}=50 \mathrm{kPa}$. However, the strains at which $\chi_{1}$ reaches its peak and $\chi_{1}$ and $\chi_{3}$ intersect both shift to the right. Furthermore, the degree of anisotropy increases with SU.

\section{Effect of Fines Content on Bishop's Coefficient}

It is commonly accepted that the internal structure of granular materials will be modified upon the addition of fine particles. For example, small amounts of silt particles introduced into sand could make the sand structure less stable, thereby increasing the compressibility of the mixture (Lade and Yamamuro 1997). The behavior of this granular mixture under external loads strongly depends on its internal force chain, through which interaction forces are transferred and endured (Thevanayagam 1998). Some fines may

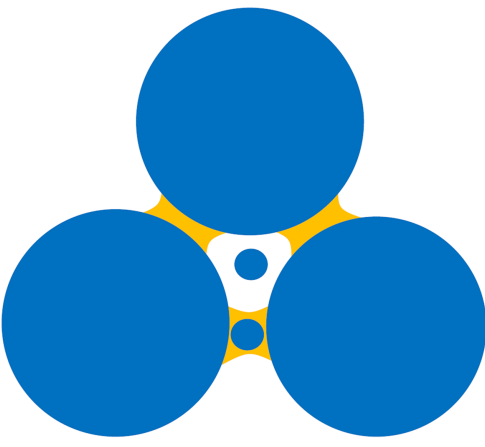

(a)

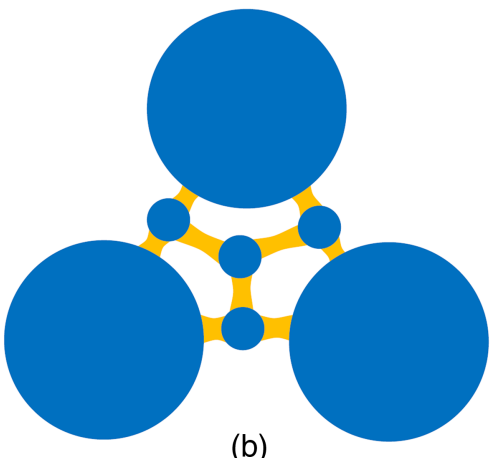

(b)

Fig. 10. Intergranular soil mix classification; network dominated by (a) coarse-grained interactions with fine particles confined within void or between grains; (b) fine-grained interactions; intergranular distances are enlarged to show network of liquid bridges

be inactive in the force transmission as they sit in voids of the soil skeleton constructed by coarse grains, while others can sustain forces as they are trapped in the contact regions between the larger grains. When more fines are added, the contact between fine grains may predominate inside the sample. An illustration of different mixture configurations is shown in Fig. 10.

In the DEM simulations conducted to investigate the effect of fines content on Bishop's coefficient $\chi$, the authors prepared a series of numerical samples composed of large particles and different amounts of fines. The same sphere packing generation algorithm as in the section "Evolution of Bishop's Coefficient during Suction-Controlled Triaxial Tests" is employed. First, for each sample, 5,000 large grains $0.1 \mathrm{~mm}$ in diameter are randomly distributed inside a cubic box made of six rigid frictionless walls with no overlaps in between. All samples have the same initial configuration of coarse grains. Then fine grains 0.04 $\mathrm{mm}$ in diameter are randomly inserted into the void of these coarse-grain networks. In the resulting mixtures, the ratios of the number of coarse grains and fine grains $n_{\text {coarse }}: n_{\text {fine }}$ are equal to $1: 7,1: 8,1: 9$, and 1:10. Each sample is consolidated closely to the same porosity of 0.342 under $10 \mathrm{kPa}$ confining pressure by setting the friction angle to as small as $0.5^{\circ}$. It is then reset to $30^{\circ}$ for a compression test along the Y-axis.

As presented in Fig. 11, $n_{\text {coarse }}: n_{\text {fine }}=1: 7$ and $n_{\text {coarse }}: n_{\text {fine }}=1: 8$ samples have nearly the same peak shear stresses as the coarse sample but possess higher residual shear stresses. The peaks in the other samples are slightly reduced, suggesting that the skeleton of coarse grains is rendered less stable by high amounts of fines. The dilatancy for all mixtures is largely increased as expected. 

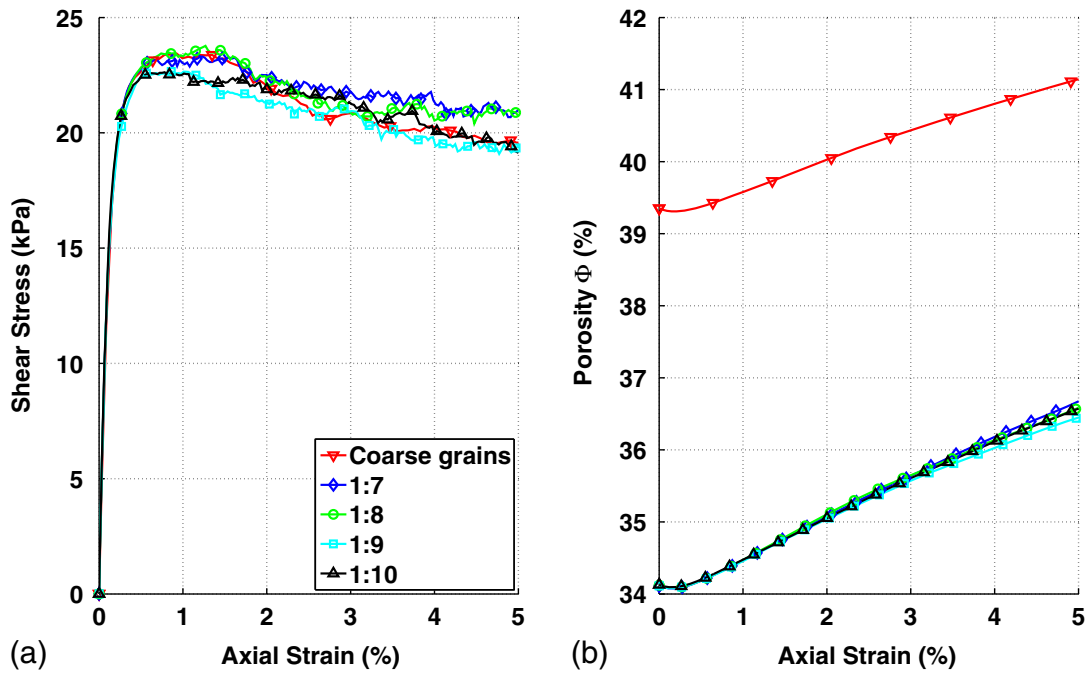

Fig. 11. (a) Shear stress and (b) porosity versus axial strain curves with $\mathrm{SU}=10 \mathrm{kPa}$ under constant confining stress $10 \mathrm{kPa}$; samples prepared with different ratios of number of coarse grains and fine grains, $n_{\text {coarse }}: n_{\text {fin }}$
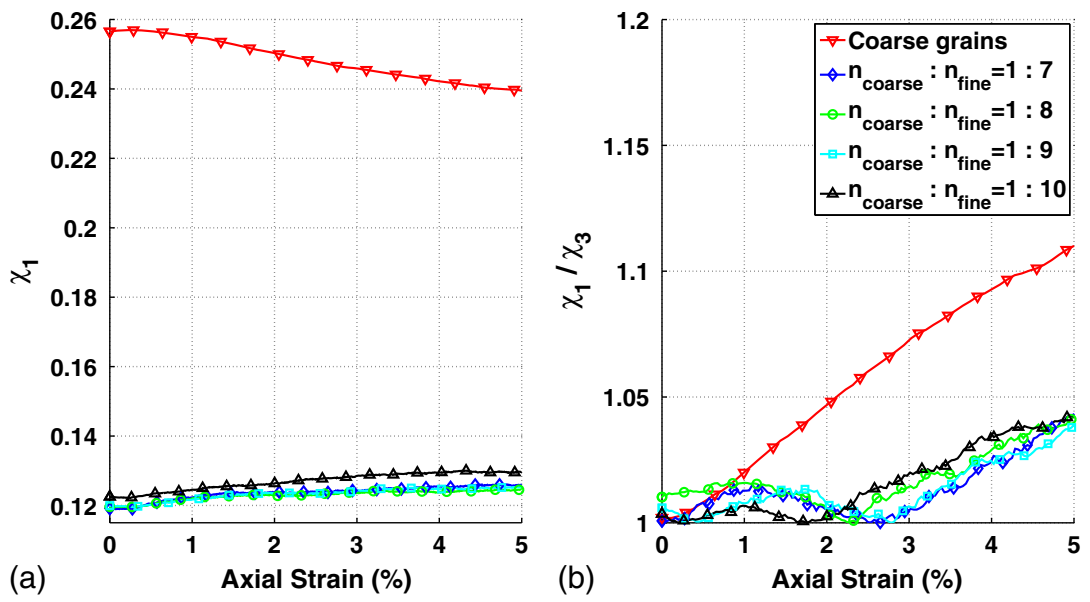

Fig. 12. (a) $\chi_{1}$ and (b) $\chi_{1} / \chi_{3}$ versus axial strain for samples of different amount ratios of coarse grains and fine grains, $n_{\text {coarse }}: n_{\text {fin }}$

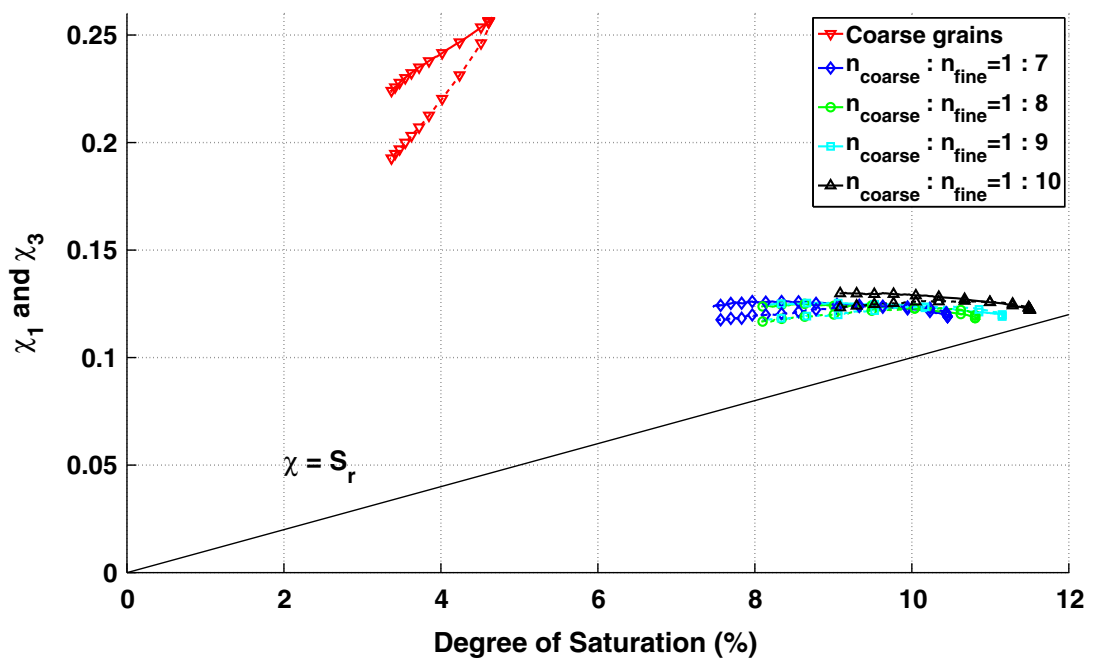

Fig. 13. $\chi_{1}$ (solid lines) and $\chi_{3}$ (dashed lines) versus degree of saturation $S_{r}$ for samples of different amount ratios of coarse grains and fine grains, $n_{\text {coarse }}: n_{\text {fin }}$ 
When different amounts of fines are added to the sample of large grains, the major principal component of Bishop's coefficient $\chi_{1}$, as well as the major/minor principal value ratio $\chi_{1} / \chi_{3}$, decreases significantly, as observed in Fig. 12. Since the initial grain assemblies composed of particles of different sizes do not necessarily yield isotropic grain structures, the principal components $\chi_{1}$ and $\chi_{3}$ may not coincide at $0 \%$ strain. For each coarse-fine grain sample in the simulations, the major principal direction corresponds to the lateral directions ( $\mathrm{X}, \mathrm{Z}$-axis) at the beginning of the deviatoric loading. As the axial strain (along Y-axis) is applied gradually, the axial component of Bishop's coefficient surpasses the lateral components, and thus the axial direction becomes the major principal direction. The strain at which the principal directions alter depends on the fines content. The $\chi_{1} / \chi_{3}$ ratio is reduced in all mixtures by approximately $6.8 \%$. This observation reveals that the mechanical response of grain assemblies with fines is more isotropic.

Fig. 13 provides the relationships between Bishop's coefficient and the degree of saturation. The increased retention of water, reduction of component values, and decreased anisotropy can be clearly observed. These phenomena make $\chi$ closer to the $\chi=S_{r}$ line, corresponding to the assumption that the coefficient is equal to the degree of saturation (Laloui and Nuth 2009).

To gain insight into how the internal structures of the coarsefine mixtures are affected by deviatoric loading and the presence of fines, the authors distinguished the contacts between two coarse grains, two fines, and coarse-fine grain pairs, then monitored the evolution of their mixtures during triaxial compression tests. The same counts were performed for liquid bridges. The results are shown in Fig. 14. It is common for coarse-coarse and coarse-fine contacts to decrease as the samples are slowly compressed along the Y-axis, while the portion of fine-fine contacts augment. Liquid bridges have similar trends, but the changes are more subtle. In addition, the researchers could see that coarse-fine interactions dominate in the $n_{\text {coarse }}: n_{\text {fine }}=1: 7$ sample. When the fines content is varied to $n_{\text {coarse }}: n_{\text {fine }}=1: 8$, the coarsefine and fine-fine occupy approximately the same portions. With
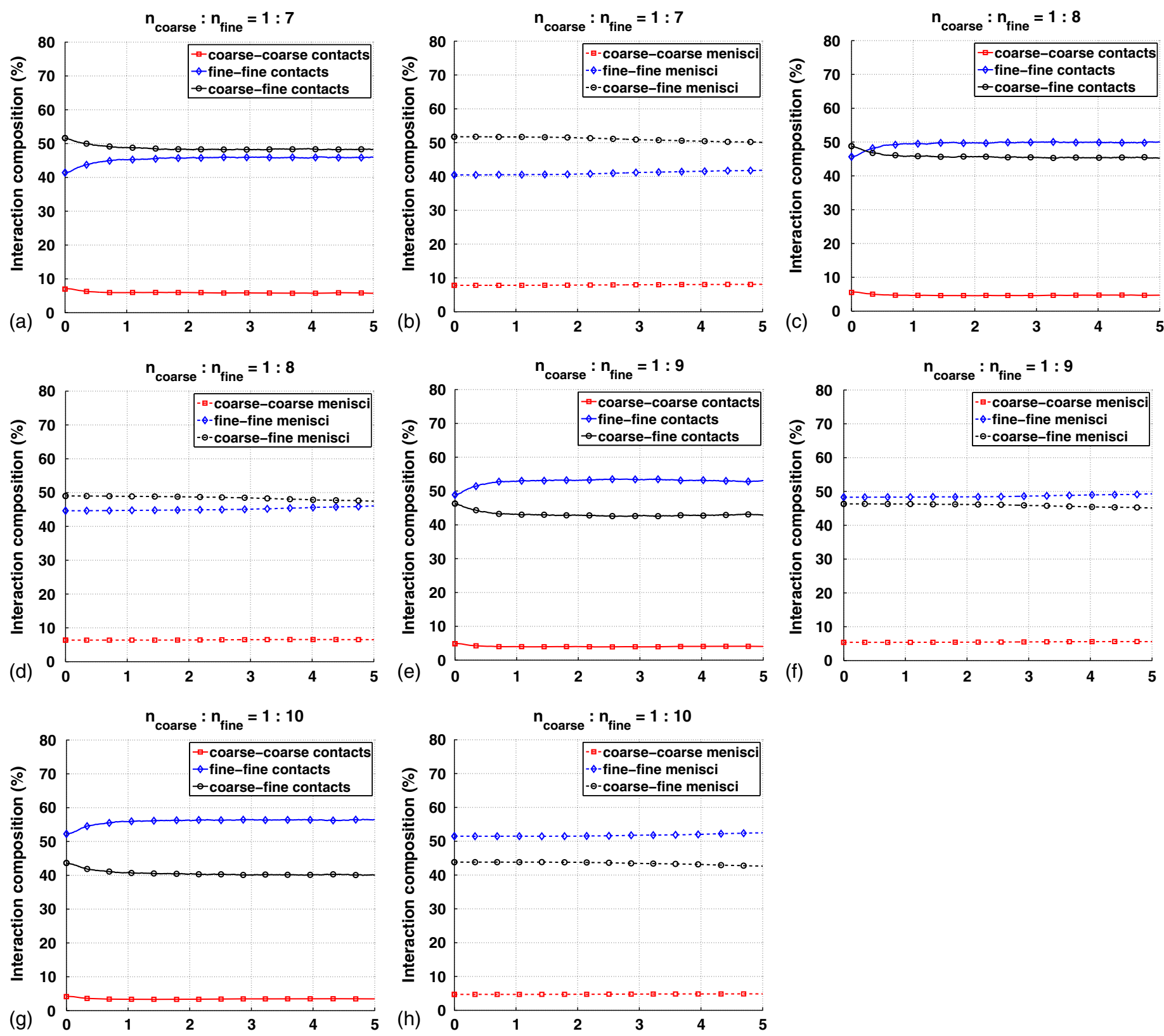

Fig. 14. Evolution of interaction compositions versus axial strain for samples of different amount ratios of coarse grains and fine grains, $n_{\text {coarse }}: n_{\text {fin }}$ 
further increases in fines content, fine-fine interactions predominate over coarse-fine interactions, indicating that the skeleton composed of fine grains starts to assume responsibility for reacting to external loads.

\section{Effect of Suction Pressure on $\chi$ for Coarse Sample and Coarse-Fine Mixture}

In this section the authors perform triaxial compression tests with different suction pressures (SU) on samples of equal-sized coarse grains $0.1 \mathrm{~mm}$ in diameter (A) and a mixture of coarse and fine grains (B). First, 5,000 large grains are distributed randomly inside a cubic box with no overlaps in between; the configuration of the grains is identical in A and B. Then, for Sample B, as many fine grains $0.04 \mathrm{~mm}$ in diameter are inserted into the void as possible. The resulting mixture has a coarse/fine grains ratio of 1:7.3 and a volume ratio of 1:0.4672. Both samples are isotropically consolidated under a confining pressure of $10 \mathrm{kPa}$ before deviatoric loading. The initial porosity of Samples A and B are 0.394 and 0.341, respectively.

Figs. 15 and 16 present the stress-strain relationships and porosity versus axial strain curves under different suction pressures of 10 , 20, and $50 \mathrm{kPa}$. Both A (left column) and B (right column) show peak shear stresses and exhibit strain-softening behavior, as well as the initial contractancy and following dilatancy. The curves suggest that adding fines to the coarse grains increases the peak shear stress and the dilatancy under the same SU. In addition, fines make the suction pressure effect more significant, based on the observation that the discrepancies in the stress and porosity curves between different pressures are enlarged for Sample B.

Figs. 17 and 18 present the evolution of principal components of Bishop's coefficient and their ratio. For Sample A, the curves of the principal value under different SUs display behavior similar to that in the study mentioned in the section "Evolution of Bishop's Coefficient during Suction-Controlled Triaxial Tests". Both $\chi_{1}$ and $\chi_{3}$ decrease as the SU increases. For Sample B, however, the evolution of the principal values changes since the initial internal structure constituted by coarse grains is modified by fines. Under a $10 \mathrm{kPa}$ suction pressure, the components significantly reduce by $54 \%$ at $0 \%$ strain compared to $\mathrm{A}$, and the component in the lateral direction is larger than the axial component the first time. The principal directions switch subsequently at approximately $2 \%$ strain. The differences in $\chi_{1}$ and $\chi_{3}$ between the cubic shape at $0 \%$ strain and the deformed shape at $5 \%$ strain also decrease. The same reduction also appears at $\mathrm{SU}=20 \mathrm{kPa}$, where the magnitudes remain the same. On the other hand, for $\mathrm{SU}=50 \mathrm{kPa}$, the initial
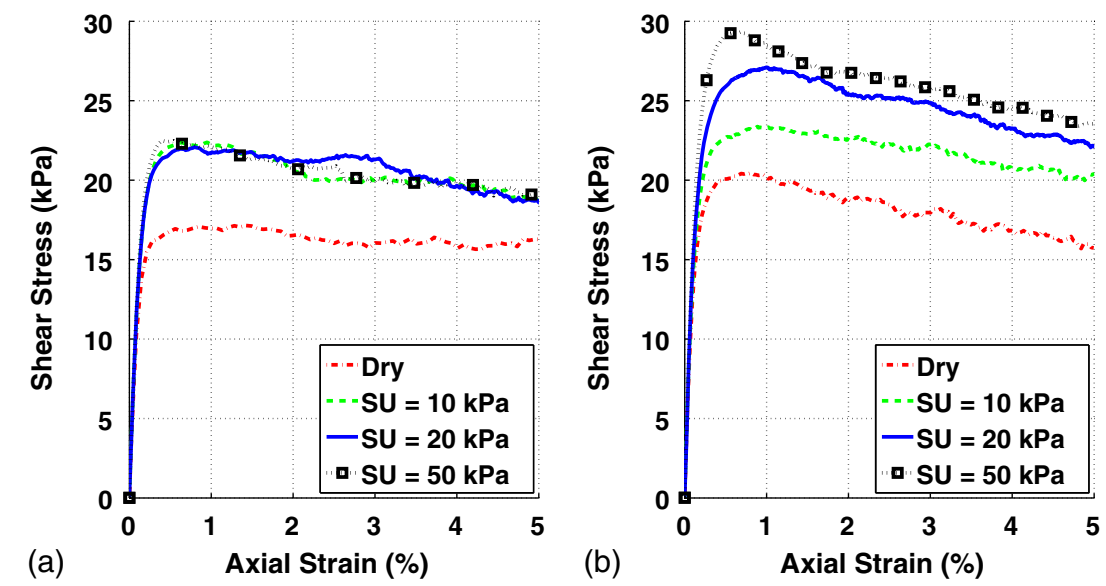

Fig. 15. Shear stress versus axial strain curves with different suctions (SU) under constant confining stress $10 \mathrm{kPa}$ : (a) sample of coarse grains; (b) mixture of coarse and fine grains
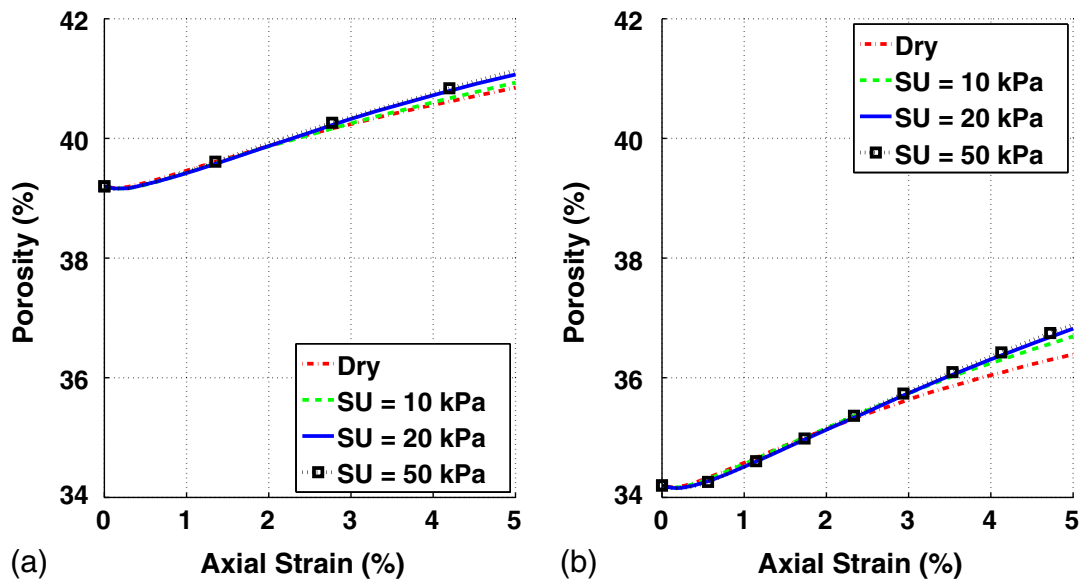

Fig. 16. Porosity versus axial strain curves with different suctions (SU) under constant confining stress $10 \mathrm{kPa}$ : (a) sample of coarse grains; (b) mixture of coarse and fine grains 

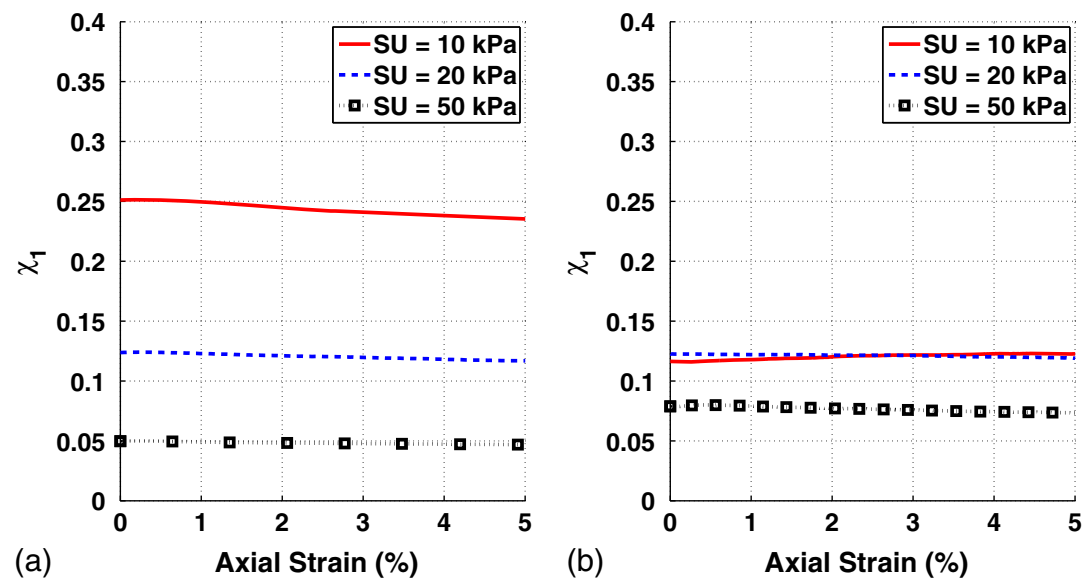

Fig. 17. (a) $\chi_{1}$ versus axial strain for sample of coarse grains and (b) mixture of coarse and fine grains
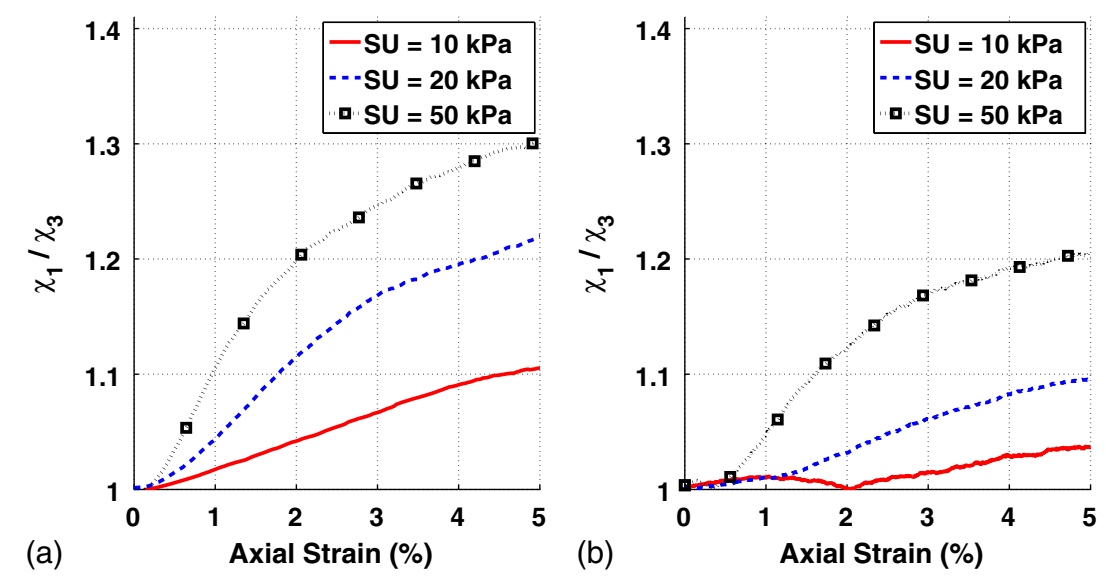

Fig. 18. (a) $\chi_{1} / \chi_{3}$ versus axial strain for sample of coarse grains and (b) mixture of coarse and fine grains
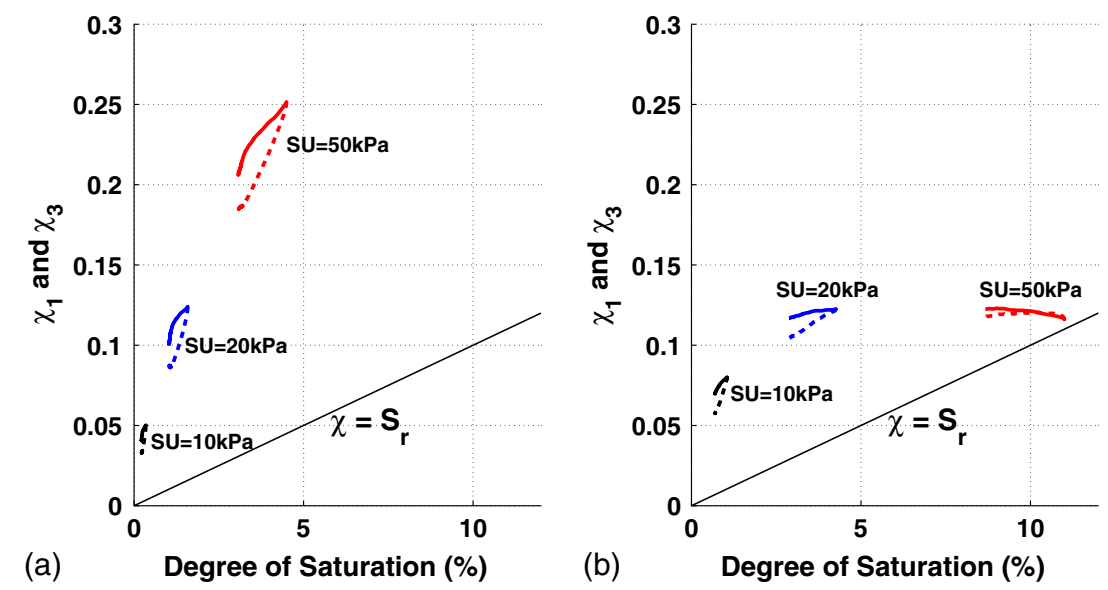

Fig. 19. (a) $\chi_{1}$ (solid lines) and $\chi_{3}$ (dashed lines) versus degree of saturation $S_{r}$ for sample of coarse grains and (b) mixture of coarse and fine grains under suction of 10,20 , and $50 \mathrm{kPa}$

values of $\chi_{1}$ and $\chi_{3}$ increase by about $58 \%$. The curves of $\chi_{1} / \chi_{3}$ in Fig. 18 indicate that the degree of anisotropy increases with SU for each sample. Comparison of A and B shows that $\chi$ becomes more isotropic when fines are added in between large particles. The $\chi_{1} / \chi_{3}$ ratio drops by $6.3,10.5$, and $7.7 \%$ for $\mathrm{SU}=10,20$, and
$50 \mathrm{kPa}$, respectively. This phenomenon may be attributed to the fact that the number of water bridges between two large grains is notably raised. Fine particles could fill the void between coarse grains and provide more proximate intergranular regions, thereby capturing much more water. Moreover, the contact normals 
between fines inside the interspace of large grains are diversified and thus make the directions of trapped menisci more random. In this case, the coarse grains are submerged in media of fines and smaller water bridges that are closer to saturated water than the discrete water bridges in Sample A. Therefore, Sample B exhibits less anisotropy on Bishop's coefficient than Sample A.

Fig. 19 describes the relationship between Bishop's coefficient and $S_{r}$ under different suction pressures. All the curves shift rightward for Sample B, confirming that more water is retained. For $\mathrm{SU}=10 \mathrm{kPa}$, the combination of this phenomenon and the reduction in the $\chi_{1}$ and $\chi_{3}$ values makes $\chi$ closer to the $\chi=S_{r}$ line.

\section{Conclusions}

In this work, the researchers introduce a tensorial Bishop coefficient $\chi$ to characterize the anisotropic responses of wetted granular materials. Using solutions from the Young-Laplace equation, the authors conduct discrete element simulations to analyze how the formation and rupture of liquid bridges affect the principal values and directions of the tensorial Bishop coefficient for two grain assemblies undergoing triaxial compression and extension loading paths. The results suggest that the anisotropy of $\chi$ is inherently path dependent and related to suction pressure. A statistical analysis of the interaction normal distribution allowed for a microscale investigation of the effect of contact and meniscus fabric evolution on Bishop's coefficient. Another group of simulations was performed on samples of coarse grains whose internal skeleton was modified by the insertion of different amounts of fine grains. These samples of various fines contents were subjected to triaxial compression loading. Results suggest a dependence of Bishop's coefficient on the type of interaction that dominates the skeleton. Comparing results for both coarse samples and mixtures, the authors concluded that the addition of fines captures more water and renders the distribution of liquid bridges less discontinuous and less anisotropic. On the other hand, the tests under different suction pressures confirmed that a sample with high SU retains less volume of water and thus has a more discrete meniscus distribution and shows a more anisotropic effect on Bishop's coefficient.

This paper focuses on the study of unsaturated granular matter in a pendular regime. To extend this study to higher degrees of saturation, the Young-Laplace equation could no longer be used. The usage of computational fluid dynamics (CFD) or two-phase flow interacting with DEM is more appropriate and will fall within the scope of future studies. The current model also does not consider factors such as surface roughness, hysteresis of contact angle, and the coalescence and redistribution of liquid bridges. The study within this paper is based on an idealized numerical model and does not fully replicate the real behavior of unsaturated soils. Ongoing research is being performed to improve the numerical model using comparisons with experimental results.

\section{Acknowledgments}

This research was partially supported by the Earth Materials and Processes program at the US Army Research Office under Grant Contract W911NF-14-1-0658 and Provost's Grants Program for Junior Faculty Who Contribute to the Diversity Goals of the University at Columbia University. These supports are gratefully acknowledged. The authors also thank the anonymous reviewers for their insightful suggestions and detailed feedback.

\section{References}

Alonso, E. E., Pereira, J.-M., Vaunat, J., and Olivella, S. (2010). A microstructurally based effective stress for unsaturated soils, Géotechnique, 60(12), 913-925.

Bishop, A., and Blight, G. (1963). Some aspects of effective stress in saturated and partly saturated soils, Geotechnique, 13(3), 177-197.

Bishop, A. W. (1959). “The principles of effective stress.” Teknisk Ukeblad, 106(39), 859-863.

Borja, R. I., and Koliji, A. (2009). "On the effective stress in unsaturated porous continua with double porosity." J. Mech. Phys. Solids, 57(8), $1182-1193$.

Cambou, B., Jean, M., and Radjaï, F. (2009). Micromechanics of granular materials, Wiley, Hoboken, NJ.

Cundall, P. A., and Strack, O. D. (1979). "A discrete numerical model for granular assemblies." Géotechnique, 29(1), 47-65.

De Boer, R. (1996). "Highlights in the historical development of the porous media theory: Toward a consistent macroscopic theory." Appl. Mech. Rev., 49(4), 201-262.

Di Renzo, A., and Di Maio, F. P. (2004). "Comparison of contact-force models for the simulation of collisions in dem-based granular flow codes." Chem. Eng. Sci., 59(3), 525-541.

Fall, A., et al. (2014). "Sliding friction on wet and dry sand." Phys. Rev. Lett., 112(17), 175-502.

Hashemi, M. A., Khaddour, G., François, B., Massart, T. J., and Salager, S. (2013). "A tomographic imagery segmentation methodology for three-phase geomaterials based on simultaneous region growing." Acta Geotech., 9(5), 1-16.

Hicher, P.-Y., and Chang, C. (2007). "A microstructural elastoplastic model for unsaturated granular materials." Int. J. Solids Struct., 44(7), 2304-2323.

Higo, Y., Oka, F., Sato, T., Matsushima, Y., and Kimoto, S. (2013). "Investigation of localized deformation in partially saturated sand under triaxial compression using microfocus $\mathrm{x}$-ray ct with digital image correlation." Soils Found., 53(2), 181-198.

Hotta, K., Takeda, K., and Iinoya, K. (1974). "The capillary binding force of a liquid bridge." Powder Technol., 10(4), 231-242.

Iwashita, K., and Oda, M. (2000). "Micro-deformation mechanism of shear banding process based on modified distinct element method." Powder Technol., 109(1), 192-205.

Jennings, J., and Burland, J. (1962). "Limitations to the use of effective stresses in partly saturated soils." Géotechnique, 12(2), 125-144.

Jiang, M., Leroueil, S., and Konrad, J. (2004). "Insight into shear strength functions of unsaturated granulates by dem analyses." Comput. Geotech., 31(6), 473-489.

Koiso, M., and Palmer, B. (2007). "Anisotropic capillary surfaces with wetting energy." Calculus Variations Partial Differ. Equations, 29(3), 295-345.

Kruyt, N., and Rothenburg, L. (1996). "Micromechanical definition of the strain tensor for granular materials." J. Appl. Mech., 63(3), 706-711.

Lade, P. V., and Yamamuro, J. A. (1997). "Effects of nonplastic fines on static liquefaction of sands." Can. Geotech. J., 34(6), 918-928.

Laloui, L., and Nuth, M. (2009). "On the use of the generalised effective stress in the constitutive modelling of unsaturated soils." Comput. Geotech., 36(1), 20-23.

Lewis, R. W., and Schrefler, B. A. (1998). The finite element method in the static and dynamic deformation and consolidation of porous media, Wiley, Chichester.

Lian, G., Thornton, C., and Adams, M. J. (1993). "A theoretical study of the liquid bridge forces between two rigid spherical bodies." J. Colloid Interface Sci., 161(1), 138-147.

Lu, N., and Likos, W. J. (2004). Unsaturated soil mechanics, Wiley, Hoboken, NJ.

Lu, N., Wu, B., and Tan, C. P. (2007). "Tensile strength characteristics of unsaturated sands." J. Geotech. Geoenviron. Eng., 10.1061/(ASCE) 1090-0241(2007)133:2(144), 144-154.

Manahiloh, K. N., and Muhunthan, B. (2012). "Characterizing liquid phase fabric of unsaturated specimens from x-ray computed tomography images." Unsaturated soils: Research and applications, Springer, Berlin. 
Richefeu, V., El Youssoufi, M. S., and Radjai, F. (2006). "Shear strength properties of wet granular materials." Phys. Rev. E, 73(5), 304.

Salager, S., Jamin, F., El Yussoufi, M. S., and Saix, C. (2006). "Influence of temperature on the water retention curve of porous media." Comptes Rendus Mecanique, 334(6), 393-398.

Scholtès, L., Hicher, P.-Y., Nicot, F., Chareyre, B., and Darve, F. (2009). "On the capillary stress tensor in wet granular materials." Int. J. Numer. Anal. Methods Geomech., 33(10), 1289-1313.

Shapiro, S. (2000). "The effects of nonplastic fines on the three-dimensional behavior of sand." Ph.D. thesis, Clarkson Univ., Potsdam, NY.

Sheng, D., Sloan, S., and Gens, A. (2004). "A constitutive model for unsaturated soils: Thermomechanical and computational aspects." Comput. Mech., 33(6), 453-465.

Shi, D., and McCarthy, J. J. (2008). "Numerical simulation of liquid transfer between particles." Powder Technol., 184(1), 64-75.

Šmilauer, V., et al. (2014). "Yade documentation." 〈http://yade-dem.org/ doc/> (Sept. 20, 2014).

Soulie, F., El Youssoufi, M. S., Cherblanc, F., and Saix, C. (2006). "Capillary cohesion and mechanical strength of polydisperse granular materials." Eur. Phys. J. E: Soft Matter Biol. Phys., 21(4), 349-357.

Sun, W. (2013). "A unified method to predict diffuse and localized instabilities in sands." Geomech. Geoeng., 8(2), 65-75.

Sun, W., Andrade, J. E., and Rudnicki, J. W. (2011a). "Multiscale method for characterization of porous microstructures and their impact on macroscopic effective permeability." Int. J. Numer. Methods Eng., 88(12), $1260-1279$

Sun, W., Andrade, J. E., Rudnicki, J. W., and Eichhubl, P. (2011b). "Connecting microstructural attributes and permeability from 3D tomographic images of in situ shear-enhanced compaction bands using multiscale computations." Geophys. Res. Lett., 38(10).

Sun, W., Chen, Q., and Ostien, J. T. (2014). "Modeling the hydro-mechanical responses of strip and circular punch loadings on water-saturated collapsible geomaterials." Acta Geotech., 9(5), 903-934.

Sun, W., Kuhn, M. R. and Rudnicki, J. W. (2013a). "A multiscale DEMLBM analysis on permeability evolutions inside a dilatant shear band." Acta Geotech., 8(5), 465-480.

Sun, W., Ostien, J. T., and Salinger, A. G. (2013b). "A stabilized assumed deformation gradient finite element formulation for strongly coupled poromechanical simulations at finite strain." Int. J. Numer. Anal. Methods Geomech., 37(16), 2755-2788.

Terzaghi, K. (1996). Soil mechanics in engineering practice, Wiley, New York.

Thevanayagam, S. (1998). "Effect of fines and confining stress on undrained shear strength of silty sands." J. Geotech. Geoenviron. Eng., 10.1061/(ASCE)1090-0241(1998)124:6(479), 479-491.

Toker, N. K., Germaine, J. T., and Culligan, P. J. (2014). "Effective stress and shear strength of moist uniform spheres." Vadose Zone J., 13(5), in press.

Wan, R., Khosravani, S., and Pouragha, M. (2014). "Micromechanical analysis of force transport in wet granular soils." Vadose Zone J., 13(5), in press.

Wheeler, S., Gallipoli, D., and Karstunen, M. (2002). "Comments on use of the barcelona basic model for unsaturated soils." Int. J. Numer. Anal. Methods Geomech., 26(15), 1561-1571.

Zienkiewicz, O. C., Chan, A., Pastor, M., Schrefler, B., and Shiomi, T. (1999). Computational geomechanics, Wiley, Chichester. 